\title{
Differential Frequency Hopping Signal Detection Based on HHT
}

\author{
Shufeng QI, Wei QUAN, Zhiwen XIAO, Qun ZHAO \\ Dalian Air Force Communication NCO Academy, Dalian Liaoning 116100, China
}

\begin{abstract}
Differential frequency hopping communication system as a new concept of communication systems has become an important development direction of the current shortwave confidential communications. In this paper, on the DFH communication mechanism based on the establishment of a function, the function design m sequences designed based on HHT transform DFH signal detection design, experimental results show that this scheme can effectively achieve DFH Signal generation and detection of a communication system.
\end{abstract}

KEYWORD: DFH; G function; HHT transform; detection

\section{INTRODUCTION}

HF communication as a strong invulnerability and low cost means of communication, in a variety of fields has been widely applied, the differential frequency hopping spread spectrum communication technology is a new technology emerged in recent years [1-3], which sets such as frequency hopping patterns, information modulation and demodulation functions, composition and frequency hopping technology is completely different from the traditional technology system.

There are short-time Fourier transform (STFT) [3-5] for the DFH signal detection method, rotation invariant subspace (ESPRIT) [6], higher-order cumulants [7] and wavelet [8-9] or the like. STFT method [3-4] DFH signal for short-time Fourier transform, extract the signal frequency analysis results, combined with functions to achieve effective DFH signal detection algorithm is relatively simple, but less time-frequency energy aggregation, the need to predict parameters such as frequency hopping point. [5] first jump frequently extracted using STFT point, re-use auto-regressive moving average (ARMA) algorithm to eliminate noise, the further integration of the least mean square algorithm detects the differential frequency hopping signals, which can be eliminated by a certain extent frequency noise caused by false energy, but need to predict DFH signal frequency parameters. [6] The ESPRIT method of frequency hopping signals to detect the difference, but need to use array antenna receiving the signal, the receiving process is more complex, large amount of calculation, again, point to predict parameters such as frequency hopping. [7] proposed slice-based transient signal detection method DFH 4-order moments of the phase information, the use of higher-order moments of the instantaneous autocorrelation function of the realization of the differential frequency hopping signals jump parameter estimates, but in an unknown signal DFH frequently point jump, the need for all frequencies of the plane slicing search, poor timeliness. [8] and [9] are based on wavelet transform to achieve the detection of differential frequency hopping signals, which the literature [8] using differential frequency hopping signal and noise signal singularity different effective detection; literature [9] first the signal using wavelet timefrequency analysis, frequency ridge line extraction, further estimates DFH signal and frequency hopping parameters, its implementation still predict effectively detect frequency hopping points.

Given the frequency hopping sequence DFH signal has no cycle characteristics, frequently point jump in unknown circumstances, the above detection method has some limitations; DFH signal and still has the characteristics of frequency changes over time, only the signal Frequency hopping can not be distinguished based on the received signal into a differential frequency hopping signals or conventional shortwave frequency hopping signals. In this paper, a method for detecting unknown signal parameters DFH signal conditions made for this study, based on the analysis of differential frequency hopping signal generation mechanism on the receiving end in Hirbert DFH signal through indepth study of GGT transformed spectrum, were 
unknown Research hopping signal detection method differential signal parameters under the conditions.

\section{BASIC PRINCIPLE OF DFH}

The concept stems from a DFH enhanced 1995 Sandes company developed Spread Spectrum (Correlated Hopping Enhanced Spread Spectrum, called CHESS) system, CHESS radio with DFH (Differential Frequency Hopping, referred DFH) as the core technology to achieve high-speed frequency hopping, hopping speed up to 5000 hops / sec transfer rate up to $19.2 \mathrm{kbit} / \mathrm{s}$, and anti-jamming, strong anti-fading capability.

DFH has two key technologies, namely signal detection technology design technology and the receiving end of a differential frequency hopping pattern.

DFH system uses a new technology system, its design is based on the hopping pattern called a mathematical relationship of $G$ function, at the sending end, the frequency value of the current time $f_{n}$ is by the frequency value $f_{n-1}$ and the current time data symbols $X_{n}$ decision. An implicit difference equation is expressed as [8]:

$$
f_{n}=G\left(f_{n-1}, X_{n}\right)
$$

$\mathrm{G}(\cdot)$ indicates a function transform relations, referred to function. Its frequency, data transformation relationship can be expressed in the form of a diagram of transformation function in Figure 1.

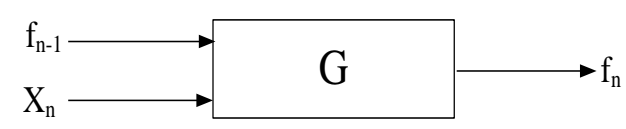

Fig.1 The $\mathrm{G}$ function transform

Differential frequency hopping communication system, the receiver uses a variety of new technology to receive broadband, digital signal processing. At the receiving end, by the broadband receiving digitized, characterized by the FFT analysis of all signals within the bandwidth of the frequency hopping, $f_{n-1}$ and $f_{n}$ is determined. Restore the data transmitting side by the inverse transform function, namely:

$$
X_{n}=G^{-1}\left(f_{n-1}, f_{n}\right)
$$

Wherein $\mathrm{G}^{-1}$ denotes inverse transform function of $G$, which requires the function $G$ must have reversible. Its inverse transform relationship can be expressed in the form of a diagram 2 .

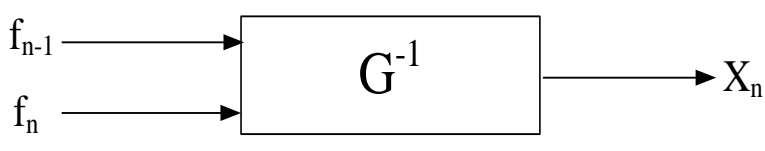

Fig.2 The inverse $\mathrm{G}$ function transform

\section{G FUNCTION ALGORITHM PRINCIPLE}

In the differential frequency hopping, $G$ function is a specific frequency transfer function, can be seen as a directed graph, directed graph node represents a frequency point of each frequency set, each node separates $f=2^{B P H}$ branches, BPH representative of the number of bits per hop transmission, also called the fan-out coefficients. Are marked on each bifurcation current information symbols to the set of frequencies containing frequencies of 64, for example, when each hop transmission 1bit, each node has two bifurcated $(0,1)$. BPH transmission bit stream bits per symbols constituting a transmission symbol stream according to a given differential frequency hopping sequence with a frequency corresponding to the generated map.

\subsection{1 bit G function design}

$\mathrm{G}$ function design approach are many, including those based on the theory of congruence $\mathrm{G}$ function design, based on the state of the shift register design function $\mathrm{G}, \mathrm{G}$ function $\mathrm{m}$-sequence-based design and so on. In this selection of designs based on $G$ function $\mathrm{m}$ sequences. Algorithm is described as follows:

Step1 First reads the data $X_{n}$ to be transmitted, the data is encoded, the encoded data $d_{n}$ is calculated.

$$
d_{n}= \begin{cases}1 & X_{n}=0 \\ -1 & X_{n}=1\end{cases}
$$

step2 Front jump frequency $F_{n-1}$, frequency hopping pattern points $\mathrm{N}$, jump into a subset of frequently point number $\mathrm{P}, \mathrm{m}$ sequence truncated after 2 bit bit $m_{1}$ and $m_{2}$, the encoded data $d_{n}$ into equation (2), find the current hop frequency.

$$
F_{n}=\left(F_{n-1}+d_{n}{ }^{2}+2 d_{n}\right) \bmod (N / p)+(N / p)\left(m_{1}+m_{2}\right)(4)
$$

Step3 Frequency $F_{n}$ is verified, if $F_{n}=F_{n-2}$, representing two same-hop, $F_{n-2}$ represents the first two jump frequency, the generation type (5) of the transfer function, $F_{n}$ is recalculated:

$$
F_{n}=\left(F_{n-2}+N / p\right) \bmod (N)
$$

Step4 Final $F_{n}$ will be stored, and then return to Step1 recalculated under the jump. 


\subsection{1 bit $G-1$ function design}

Combined with the above 1 bit $G$ function design algorithm, we propose a fast algorithm for seeking $\mathrm{G}^{-1}$ function, the algorithm is described as follows:

Stepl Read detected two consecutive jump frequency $F_{n}$ and $F_{n-1}$, by the formula (6) determine the frequency subscript value $\Delta \varepsilon$.

$\Delta \varepsilon=F_{n}-F_{n-1}$

Step2 Intermediate calculation value $\Delta H$ of the function is calculated according to equation (7) and related parameters of $\mathrm{G}^{-1}$ function.

$$
\Delta H=\left\{\begin{array}{lc}
\Delta \varepsilon & |\Delta \varepsilon| \leq 3 \\
\Delta \varepsilon-N / p & 3<\Delta \varepsilon \leq N / p+3 \\
\Delta \varepsilon-2 N / p & N / p+3<\Delta \varepsilon \leq 2 N / p+3 \\
\Delta \varepsilon-3 N / p & 2 N / p+3<\Delta \varepsilon \leq 3 N / p+3 \\
\Delta \varepsilon-N & 3 N / p+3<\Delta \varepsilon \leq N-1 \\
\Delta \varepsilon+N / p & 3<-\Delta \varepsilon \leq N / p+3 \\
\Delta \varepsilon+2 N / p & N / p+3<-\Delta \varepsilon \leq 2 N / p+3 \\
\Delta \varepsilon+3 N / p & 2 N / p+3<-\Delta \varepsilon \leq 3 N / p+3 \\
\Delta \varepsilon+N & 3 N / p+3<-\Delta \varepsilon \leq N-1
\end{array}\right\}
$$

Step3 $\Delta H$ will get correspondence in Table 1, the data $X_{n}$ will be decoded by demodulation function.

Table $1 \mathrm{G}$ function decoding function correspondence tables

\begin{tabular}{|c|c|c|c|c|}
\hline$\Delta H$ & 3 & 1 & -1 & -3 \\
\hline$X_{n}$ & 0 & 0 & 1 & 1 \\
\hline
\end{tabular}

Step4 Finally storing the demodulated data $X_{n}$, and then returns to recalculate Step1

\section{SIGNAL DETECTION BASED ON HHT}

\subsection{The basic principle of HHT algorithm}

1998 Norden E. Huang et al proposed Hilbert-Huang Transform (HHT), the method is particularly suited to deal with non-stationary signals. Currently, HHT method has been successfully applied in many fields of geophysics, biomedical and fault detection, reflects its unique advantages. HHT method is mainly divided into two parts, the first signal is decomposed into a finite number of intrinsic mode functions (Intrinsic Mode Function, IMF), then for each IMF Hilbert transform decomposition, the final summary of all the IMF Hilbert transform to obtain the original signal time-frequency plane energy distribution spectra, namely Hilbert spectrum, Hilbert spectrum can accurately portray the signal energy over time, the distribution of frequencies that can be made precise time - frequency - energy diagram.

\subsection{DFH signal generator}

Assuming DFH signal jump speed 5000Hop / s, the frequency hopping pattern of points $\mathrm{N}=64$, the difference in the $3.15 \mathrm{MHz}$ frequency hopping signal range hopping, hopping DFH simulation signal bandwidth between $13.25 \mathrm{MHz} \sim 16.4 \mathrm{MHz}$, the frequency control word and frequency of correspondence between DFH signal as shown in Table 2.

Table 2 Frequency control word and frequency of DFH signal corresponding relationship

\begin{tabular}{|l|c|c|c|c|c|}
\hline $\begin{array}{l}\text { Frequency control } \\
\text { word }\end{array}$ & 0 & 1 & $\ldots$ & 62 & 63 \\
\hline Frequency / MHz & 13.25 & 13.3 & $\ldots$ & 16.35 & 16.4 \\
\hline
\end{tabular}

According to 1 bit $\mathrm{G}$ function design scheme, simulates simulated DFH signals.

\subsection{DFH signals detection based on Hilbert spectrum}

At the receiving end, the received signal HHT transform, Hilbert spectrum frequency focus of good use when the detection signal frequency. DFH previous three jump signal receiving and processing process, for example, after HHT transform, frequency-point detection, the results shown in Figure 3.

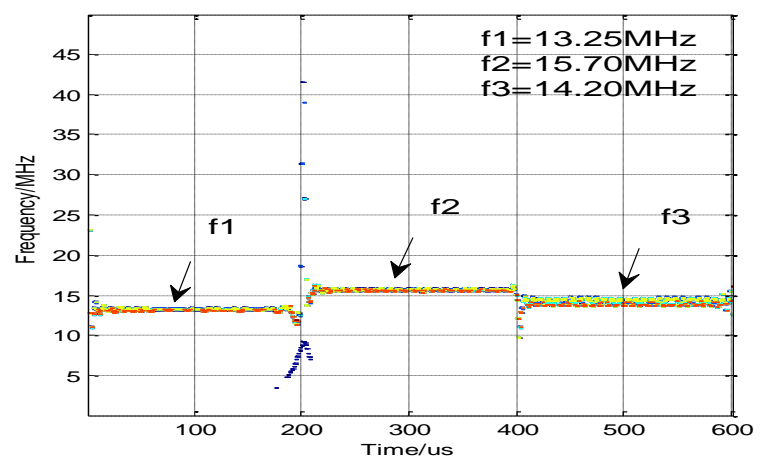

Fig.3 The result of HHT detection

Test results can be seen from Figure 3, the first three jump frequencies were $13.25 \mathrm{MHz}, 15.7 \mathrm{MHz}$ and 14.2MHZ, according to the $\mathrm{G}^{-1}$ function, data can be received, and DFH analog signal generated by the transmitter match proved the effectiveness of HHT detection method.

\section{SUMMARY}

DFH technology is a new communications technology system, has many excellent properties, is one of the future direction of development of communication technology. In this paper, the function $\mathrm{m}$ sequences generated DFH analog signal at the receiving end, the use of HHT transform, the 
received signal is detected, the experimental results show that the structure of the m-sequence function can generate a differential frequency hopping signals, HHT transform can DFH effective detection signal.

\section{REFERENCES}

[1] Chen z, Li S Q. Performance of differential frequency hopping receivers over Rayleigh-fading channel with multitone jamming //communications circuits and systems IEEE international conference. 2009: 147-151.

[2] Zhao Liping, Yao Fuqiang, Li Yongguai. The making-up of differential frequency hopping network with its characteristics. Acta electronica sinica, 2006, 34(10):18881891.

[3] Liu Zhaongying, Zhang Yi, Yao Fuqiang. HF DFH hopdetecting method based on STFT and G function. Acta electronica sinica, 2003, 31(11):1-5.
[4] Jin Qiang, Lou Caiyi. Piolt study of detection and jamming of CHESS radio. Communication countermeasures, 2009, 24(1):33-36.

[5] Sui D, Ge L D. A new frequency estimation approach to DFH signal with periodgram and ARMA //information communications and signal processing fifth IEEE international conference. 2005:739-742.

[6] Zhang zhiheng, Wu jie. Research on application of ESPRIT algorithm in CHESS frequency detection. Communications technology, 2008, 41(10): 29-31.

[7] Tuan zili, He zunwen. A detection method of DFH signals. Ship science and technology, 2009, 31(1):86-89.

[8] Wang minghai, Gou yangxin. A differential frequency hopping signal detection method based on wavelet trans form and time-frequency analysis. Telecommunication engineering, 2008, 48(3): 86-90.

[9] Zhou yunwei, Zhao rongli. Existence detection of DFH signals. Journal of the china railway society, 2002, 24(3): 40-44. 